\title{
THE JOSIAH WILLARD GIBBS LECTURESHIP
}

The Council of the Society has sanctioned the establishment of an honorary lectureship to be known as the Josiah Willard Gibbs Lectureship. The lectures are to be of a popular nature on topics in mathematics or its applications, and are to be given by invitation under the auspices of the Society. They will be held annually or at such intervals as the Council may direct. It is expected that the first lecture will be delivered in New York City during the winter of 1923-24, and a committee has been authorized to inaugurate the lectures by choosing the first speaker and making the necessary arrangements.

R. G. D. Richardson, Secretary.

\section{AN ELECTROMAGNETIC THEORY OF LIGHT-DARTS}

BY H. BATEMAN

1. Introduction. Since Einstein's theory of light-quanta * is being carefully tested in many ways by ingenious experiments and is gaining favor, it is thought by some physicists that in the future Maxwell's equations will no longer be regarded as the best starting point for the elucidation of physical phenomena but will be derived from the equations of quantum-theory by some limiting process.

On the other hand, it may be argued that physicists have been led to this point of view because they refuse to believe what is clearly indicated by some of the most interesting solutions of Maxwell's equations. These solutions show that

* A. Einstein, Annalen der Physik, vol. 17 (1905), p. 132; PhysikalISCHe Zeitschrift, vol. 10 (1909), p. 185; vol. 18 (1917), p. 121. 
light-darts * of an electrical nature may have a real existence and that it is not impossible for electricity to travel with the velocity of light.

The chief difficulty in developing an electromagnetic theory of light-darts is that of the location of the energy of a lightdart. It is not certain that we can speak of a definite location of energy. Thus in electrostatics the energy can be expressed either as an integral of type $\dagger \frac{1}{2} \mathcal{S} V d e$ taken over the electric charges, or as an integral over the whole of space in which the density of energy is proportional to the square of the force.

The latter expression is usually preferred in forming a physical picture because the density of energy cannot be negative, while there is just a possibility that $V$ and de might have opposite signs. If, however, this possibility is excluded $\ddagger$ we can regard the energy either as spread throughout the whole of space or as localized in the charges. The structure of the universe may, indeed, be such that it may be pictured in more than one way, and it is quite possible that electromagnetic theory and the theory of quanta are two different methods of calculation which will prove eventually to be equivalent, though different distributions of energy are considered in the two cases.

2. Field Travelling in One Direction. To make this point of view clear, let us consider an electromagnetic field specified by field-vectors $E$ and $H$ with components

(1) $\left\{\begin{array}{lll}E_{x}=0, & E_{y}=\frac{\partial \Omega}{\partial y} f\left(t-\frac{x}{c}\right), & E_{z}=\frac{\partial \Omega}{\partial z} f\left(t-\frac{x}{c}\right), \\ H_{x}=0, & H_{y}=-\frac{\partial \Omega}{\partial z} f\left(t-\frac{x}{c}\right), & H_{z}=\frac{\partial \Omega}{\partial y} f\left(t-\frac{x}{c}\right),\end{array}\right.$

* The term light-dart is recommended by Silberstein, PHILosophical Magazine, vol. 44 (1922), pp. 257, 956.

$\dagger$ In this integral $V$ represents the electrostatic potential and de an element of charge.

$\ddagger V$ and $d e$ will always have the same sign if charges of opposite signs are kept far enough apart and a large number of charges of one sign are never close to one of the opposite sign. 
where $\Omega$ is a function of $y$ and $z$ representing the logarithmic potential of a distribution of line charges bounded by a cylindrical surface $\psi(y, z)=0$. We may thus write

$$
\left\{\begin{aligned}
\frac{\partial^{2} \Omega}{\partial y^{2}}+\frac{\partial^{2} \Omega}{\partial z^{2}} & =0, & & \psi(y, z)>0 \\
& =\sigma(y, z), & & \psi(y, z)<0 .
\end{aligned}\right.
$$

It is easily seen that the fundamental equations of the theory of electrons are satisfied with the above expressions for the components of $E$ and $H$ if the electricity in the field has a volume density $\rho$ given by

$$
\left\{\begin{array}{lll}
\rho=0, & \text { if } & \psi(y, z)>0, \\
\rho=\sigma(y, z) f\left(t-\frac{x}{c}\right), & \text { if } & \psi(y, z)<0,
\end{array}\right.
$$

and moves with the velocity of light in the direction of the axis of $x$. The energy in the field is

$$
\begin{aligned}
\frac{1}{2} \mathcal{S}\left(E^{2}+H^{2}\right) & d x d y d z \\
& =\int\left[\left(\frac{\partial \Omega}{\partial y}\right)^{2}+\left(\frac{\partial \Omega}{\partial z}\right)^{2}\right]\left[f\left(t-\frac{x}{c}\right)\right]^{2} d x d y d z \\
& =-\int \Omega \sigma\left[f\left(t-\frac{x}{c}\right)\right]^{2} d x d y d z
\end{aligned}
$$

where the latter integral is taken over the charges in the field, the integrals

$$
\int\left[\left(\frac{\partial \Omega}{\partial y}\right)^{2}+\left(\frac{\partial \Omega}{\partial z}\right)^{2}\right] d y d z \quad \text { and } \quad-\int \Omega \sigma d y d z
$$

being equal on account of the above-mentioned property of an electrostatic field if we suppose that the integral $\int \sigma d y d z$ is zero.

Regarding the field $(E, H)$ as representing waves of light and the electricity in the field as an accompanying light-dart composed of electric doublets, we can say that the energy can be regarded either as located in the field or as associated entirely with the light-dart. It should be noticed that the energy may be finite, although electricity moves with the velocity of light. Moreover, when $\sigma(y, z)$ is positive $\Omega$ will 
be negative, if charges of opposite signs are not too close together, and each element of the integral (4) will be positive.*

3. First Example of a Field Radiating in All Directions. The field just considered is a limiting case of several types of radiant field of a more general nature. As a first example, let us take the field

$$
\left\{\begin{array}{c}
E_{x}=-\frac{X Z}{R P^{2}} f^{\prime}\left(t-\frac{R}{c}\right), \quad E_{y}=-\frac{Y Z}{R P^{2}} f^{\prime}\left(t-\frac{R}{c}\right), \\
E_{z}=\frac{1}{R} f^{\prime}\left(t-\frac{R}{c}\right), \\
H_{x}=\frac{Y}{P^{2}} f^{\prime}\left(t-\frac{R}{c}\right), \quad H_{y}=-\frac{X}{P^{2}} f^{\prime}\left(t-\frac{R}{c}\right), \\
H_{z}=0,
\end{array}\right.
$$

where

$$
\begin{gathered}
X=x-\xi, \quad Y=y-\eta, \quad Z=z-\zeta, \\
P^{2}=X^{2}+Y^{2}, \quad R^{2}=X^{2}+Y^{2}+Z^{2},
\end{gathered}
$$

and where $f(\tau)$ is an arbitrary function of $\tau, f^{\prime}(\tau)$ being used to denote its derivative with respect to $\tau$.

The point $Q$ with coordinates $(\xi, \eta, \zeta)$ is a primary singularity from which secondary singularities, carrying electric charges, are radiated with the velocity of light in directions parallel to the axis of $z$.

Take a series of such points $Q$ on a sphere of radius $a$ with center at the origin, and let us find the mean values of $E$ and $H$ for points $Q$ on this sphere.

Using a well known theorem relating to the mean value of a wave-function over the surface of a sphere $\dagger$ we find that

* It may be remarked also that the whole momentum may be regarded as associated with the light-dart. It is in the direction of the axis of $x$ and its magnitude may be derived from that of the energy by dividing by $c$. The amount of energy associated with a light-dart will depend, of course, on the properties of the atom from which the energy was radiated and there is no reason why it should not be equal to $h \nu$, where $\nu$ is the frequency associated with the function $f$ and $h$ is Planck's constant.

† See Rayleigh's Sound, vol. II, Appendix. 
at points outside the cylinder $x^{2}+y^{2}=a^{2}$, the mean values of the field-vectors have components

(6)

$$
\left\{\begin{array}{c}
\bar{E}_{x}=-\frac{x z}{r p^{2}} F\left(t-\frac{r}{c}\right), \quad \bar{E}_{y}=-\frac{y z}{r p^{2}} F\left(t-\frac{r}{c}\right), \\
\bar{E}_{z}=\frac{1}{r} F\left(t-\frac{r}{c}\right), \\
\bar{H}_{x}=\frac{y}{p^{2}} F\left(t-\frac{r}{c}\right), \quad \bar{H}_{y}=-\frac{x}{p^{2}} F\left(t-\frac{r}{c}\right), \\
\bar{H}_{z}=0, \\
p^{2}=x^{2}+y^{2}, \quad r^{2}=x^{2}+y^{2}+z^{2},
\end{array}\right.
$$

where

$$
F\left(t-\frac{r}{c}\right)=\frac{c}{2 a}\left[f\left(t-\frac{r}{c}+\frac{a}{c}\right)-f\left(t-\frac{r}{c}-\frac{a}{c}\right)\right] .
$$

The mean values of $E$ and $H$ inside the cylinder are more difficult to find but we shall not need them.

The mean values for concentric spheres within the first one can be found in the same way, and we may infer that for a radially symmetric distribution of primary singularities inside the first sphere, the field outside the cylinder $x^{2}+y^{2}=a^{2}$ is given by components of the above type but with a different function $F$.

The density of energy in the field is

$$
\frac{1}{p^{2}}\left[F\left(t-\frac{r}{c}\right)\right]^{2},
$$

and so the field energy outside the cylinder is given by the expression

$$
2 \pi \int_{0}^{\pi} \frac{d \theta}{\sin \theta} \int_{a \cos \theta}^{\infty}\left[F\left(t-\frac{r}{c}\right)\right]^{2} d r
$$

Let

$$
\left[F\left(t-\frac{r}{c}\right)\right]^{2}=-c \frac{d}{d r} \Phi\left(t-\frac{r}{c}\right),
$$

where $\Phi(-\infty)=0$; then the integral, which we shall call $I$, takes the form 


$$
I=2 \pi c \int_{0}^{\pi} \frac{d \theta}{\sin \theta} \Phi\left(t-\frac{a}{c \sin \theta}\right) .
$$

Integrating by parts, we find that *

$$
I=-2 \pi a \int_{0}^{\pi} \frac{\cos \theta}{\sin ^{2} \theta} \log \left(\tan \frac{\theta}{2}\right)\left[F\left(t-\frac{a}{c \sin \theta}\right)\right]^{2} d \theta .
$$

By putting $z=a \operatorname{ctn} \theta$, this integral may be transformed into an integral taken over the boundary of the cylinder, and finally into an integral over the region inside the cylinder. Hence it follows that the whole energy can be expressed as an integral taken over the region occupied by the electric charges, and so can be associated with the light-dart.

4. Second Example of a Field Radiating in All Directions. The field (5) is a particular case of a more general type of field in which the primary singularity $(\xi, \eta, \zeta)$ moves in an arbitrary manner with velocity less than $c$ and the direction of projection of the electric charges forming the light-darts varies with the time.

By superposing different fields of this general type other types of field may be derived by a process analogous to differentiation. The following type of field is of special interest:

Let $\tau$ be defined in terms of $x, y, z$ and $t$ by the equation $[x-\xi(\tau)]^{2}+[y-\eta(\tau)]^{2}+[z-\zeta(\tau)]^{2}=c^{2}(t-\tau)^{2}, \quad \tau \leqq t$, and let

$$
\left\{\begin{array}{c}
H_{x}=\frac{\partial(\sigma, \tau)}{\partial(y, z)}, \quad H_{y}=\frac{\partial(\sigma, \tau)}{\partial(z, x)}, \quad H_{z}=\frac{\partial(\sigma, \tau)}{\partial(x, y)} \\
E_{x}=\frac{1}{c} \frac{\partial(\sigma, \tau)}{\partial(x, t)}, \quad E_{y}=\frac{1}{c} \frac{\partial(\sigma, \tau)}{\partial(y, t)}, \quad E_{z}=\frac{1}{c} \frac{\partial(\sigma, \tau)}{\partial(z, t)}, \\
\sigma=\frac{U^{\prime}}{U}, \quad U=l X+m Y+n Z-c p T \\
U^{\prime}=l^{\prime} X+m^{\prime} Y+n^{\prime} Z-c p^{\prime} T
\end{array}\right.
$$

\footnotetext{
* The flow of momentum may be represented by a similar integral with $(1 / c) \cos ^{2} \theta$ instead of $\cos \theta$, for we need consider only the $z$-component of momentum, the $x$ - and $y$-components of the total flow of momentum being zero by symmetry. It should be noticed that each element of $I$ is positive.
} 
where

$$
X=x-\xi, \quad Y=y-\eta, \quad Z=z-\zeta, \quad T=t-\tau,
$$

and where $l, m, n$ and $p$ are functions of $\tau$ connected by the relation

$$
l^{2}+m^{2}+n^{2}=p^{2} .
$$

The symbols $l^{\prime}, m^{\prime}, n^{\prime}, p^{\prime}$ are used to denote the derivatives of $l, m, n$ and $p$ with respect to $\tau$.

The field thus specified has a primary singularity which moves according to the law

$$
x=\xi(\tau), \quad y=\eta(\tau), \quad z=\zeta(\tau) .
$$

From this primary singularity a secondary singularity is projected in the direction specified by the direction cosines $(l / p, m / p, n / p)$, and a field radiates from the different positions of the primary singularity. The portion of the field associated with any range of values of $\tau$ vanishes completely when $l, m$, $n$ and $p$ are constant for this range of values of $\tau$. If the direction of the singular ray oscillates as time varies, the light-dart will assume a wavy form and will possess a certain type of polarization.

It is easily seen that $E$ and $H$ are perpendicular and equal in magnitude while the density of energy is found to be

$$
\frac{c^{2} T^{2}}{U^{2}} \frac{l^{\prime 2}+{m^{\prime}}^{2}+{n^{\prime}}^{2}-{p^{\prime 2}}^{2}}{\left(\xi^{\prime} X+\eta^{\prime} Y+\zeta^{\prime} Z-c^{2} T\right)^{2}}=\frac{{l^{2}}^{2}+{m^{\prime}}^{2}+{n^{\prime}}^{2}-{p^{\prime}}^{2}}{c^{2}(l x+m y+n z-p r)^{2}},
$$

when

$$
\xi=\eta=\zeta=0 .
$$

When we consider a number of such fields whose primary singularities are spread, with radial symmetry, ${ }^{*}$ over a sphere of radius $a$, so that the singular rays at each instant all point in the same direction, we obtain a total field of the same

* The primary singularities are now taken to be stationary, so that $\xi^{\prime}, \eta^{\prime}$, and $\zeta^{\prime}$ are zero. 
general nature as (8) for points outside a certain tubular * surface $S$. The function $p$ may, however, have a different value. The energy outside $S$ is now expressed by the integral

$$
I=\frac{1}{c^{2}} \int_{0}^{\pi} \sin \theta d \theta \int_{r(\theta)}^{\infty} d r \int_{0}^{2 \pi} d \phi \frac{l^{\prime 2}+m^{\prime 2}+{n^{\prime 2}}^{2} p^{\prime 2}}{(1-\cos \omega)^{2}},
$$

where $\omega$ denotes the angle between the radius specified by the polar coordinates $(\theta, \phi)$ and the singular ray corresponding to the value $t-r / c$ of $\tau$.

To obtain an integrable case let us write

$$
l=p \sin \alpha \cos \beta, \quad m=p \sin \alpha \sin \beta, \quad n=p \cos \alpha,
$$

where $\alpha$ is constant and $\beta$ is a function of $t-r / c$. We may now write

$$
\cos \omega=\cos \alpha \cos \theta+\sin \alpha \sin \theta \cos (\phi-\beta) .
$$

Integrating with respect to $\phi$, we obtain

$$
I=\frac{2 \pi}{c^{2}} \int_{0}^{\pi} \sin \theta d \theta \int_{r(\theta)}^{\infty} d r \frac{p^{2}{\beta^{\prime}}^{2} \sin ^{2} \alpha(1-\cos \alpha \cos \theta) .}{(\cos \alpha-\cos \theta)^{3}} .
$$

We may now integrate with respect to $r$ by writing

where

$$
p^{2}{\beta^{\prime}}^{2}=\psi^{\prime}\left(t-\frac{r}{c}\right)
$$

We thus obtain

$$
\psi(-\infty)=0 \text {. }
$$

$$
I=\frac{2 \pi \sin ^{2} \alpha}{c} \int_{0}^{\pi} \sin \theta d \theta \frac{1-\cos \alpha \cos \theta}{(\cos \alpha-\cos \theta)^{3}} \psi\left[t-\frac{r(\theta)}{c}\right],
$$

where $r(\theta)$ is the value of $r$ on the boundary of the tubular surface, which we assume to be a surface of revolution around the axis of $z$. Integrating by parts with respect to $\theta$, we obtain an integral involving the original function $p^{2} \beta^{\prime 2}$. This integral

* The cross section of the tube will increase with the distance from the origin. It will be convenient to regard the tube as a surface of revolution; it will then not generally be completely filled with electric charges, but this does not matter. 
$\frac{2 \pi \sin ^{2} \alpha}{c} \int_{0}^{\pi} p^{2}{\beta^{\prime}}^{2} d \theta\left[\frac{1}{2} \frac{\sin ^{2} \alpha}{(\cos \alpha-\cos \theta)^{2}}+\frac{\cos \alpha}{\cos \alpha-\cos \theta}\right] \sin \theta$

consists only of positive terms, since $\alpha<\theta$, and it may be regarded as an integral over the boundary of the tubular surface. Thus the energy outside the tubular surface can be expressed as an integral over the boundary of the tubular surface. This means that the whole of the field energy can in one sense be regarded as located within a narrow region.

5. Conclusion. There may, of course, be a difference of opinion as to the physical interpretation of these results. A point of view that seems fairly reasonable is that the energy calculated is really in the field when the light-dart produces a field, but is in the light-dart when the light-dart fails to produce a field owing to the absence of ether-particles in the immediate neighborhood of the primary singularity when the light-dart is being emitted from it. The mathematical expressions for the field-vectors indicate in fact that the field is produced at the primary singularity, but they are based on the assumption that the ether is a continuous medium, whereas in reality the ether is probably discontinuous and made up of entities analogous to light-darts. Quite possibly it is only those light-darts which fail to produce fields and escape collisions during emission that have sufficient available energy to produce the photoelectric effect.

This investigation can be regarded as belonging to that class of researches in which the idea of a definite location of energy is discussed by considering the possibility of an additive distribution of stress, energy, and momentum, in which the total energy and momentum are zero and a radiation of positive energy in some directions is balanced by a radiation of negative energy in other directions, either at the same time or at a slightly different time. One type of tensor specifying such a distribution is easily written down. (See Messenger of Mathematics, vol. 52 (1922), p. 125.)

California Institute of Technology 